02

\title{
Измерения зеемановско-сверхтонкого псевдовырожденного квадруплета в $\mathrm{CaF}_{2}: \mathrm{Ho}^{3+}$
}

\author{
(C) Kieran M. Smith ${ }^{1,2,3}$, Michael F. Reid ${ }^{1,2}$, Jon-Paul R. Wells ${ }^{1,2}$ ฯ \\ ${ }^{1}$ School of Physical and Chemical Sciences, University of Canterbury, PB 4800, \\ 8140 Christchurch, New Zealand \\ ${ }^{2}$ The Dodd-Walls Centre for Photonic and Quantum Technologies, \\ New Zealand \\ ${ }^{3}$ Research School of Physics and Engineering, The Australian National University, \\ 0200 Canberra, Australia \\ e-mail: ` mike.reid@canterbury.ac.nz, ฯฯ jon-paul.wells@canterbury.ac.nz
}

Поступила в редакцию 23.07.2021 г.

В окончательной редакции 28.08.2021 г.

Принята к публикации 30.08.2021 г.

Сообщается о зеемановской инфракрасной спектроскопии электронно-ядерных уровней в переходах ${ }^{5} I_{8} \rightarrow{ }^{5} I_{7}$ в ионе $\mathrm{Ho}^{3+}$, находящемся в центре $C_{4 v}\left(\mathrm{~F}^{-}\right)$кристалла $\mathrm{CaF}_{2}$, помещенного в магнитное поле, направленное вдоль оси $\langle 111\rangle$ кристалла. Переходы в нижайшее состояние в штарковском мультиплете ${ }^{5} I_{7}$, в изолированный электронный дублет и следующую группу состояний, а именно, псевдоквадруплет, состоящий из дублета и двух соседних синглетов, демонстрируют сильно нелинейные зеемановские расщепления и вариации интенсивности. Смоделированные спектры, основанные на анализе по теории кристаллического поля, дают отличное приближение к экспериментальным данным, демонстрируя сильную предсказательную способность метода параметризованного кристаллического поля. Также предсказаны антипересечения сверхтонких подуровней, которые являются основой для хранения квантовой информации в диэлектриках, легированных редкоземельными элементами.

Ключевые слова: гольмий, редкая земля, Зееман, спектроскопия, кристаллическое поле.

DOI: $10.21883 /$ OS.2022.01.51887.40-21

\section{Введение}

Приятно отметить 80-летие профессора Марины Поповой, представив исследование сильно нелинейных зеемановских взаимодействий в сложной структуре электронно-ядерных подуровней иона $\mathrm{Ho}^{3+}$ в кристалле $\mathrm{CaF}_{2}$. Мы считаем, что эта работа уместна, потому что исследовательская группа профессора М. Поповой входит в число мировых лидеров в области спектроскопии сверхтонкой структуры уровней редкоземельных ионов, а также потому что она основана на в высшей степени плодотворном сотрудничестве в прошлом [1].

Кристаллы, легированные ионами редкоземельных элементов, являются многообещающими кандидатами для применения в устройствах квантовой информатики. Так, с использованием магнитной сверхтонкой структуры ионов $\mathrm{Eu}^{3+}$ в кристалле $\mathrm{Y}_{2} \mathrm{SiO}_{5}$ было продемонстрировано когерентное хранение в течение нескольких часов [2,3]. Время когерентности более одной секунды наблюдалось в кристалле $\mathrm{Er}^{3+}: \mathrm{Y}_{2} \mathrm{SiO}_{5}$, помещенном в магнитное поле 7T [4]. Большое время когерентности, зарегистрированное в работе [2], было получено с использованием перехода на уровень с нулевым в первом порядке эффектом Зеемана (переход ZEFOZ или „часовой“), который нечувствителен к небольшим магнитным флуктуациям. Такие переходы обычно связаны с антипересечениями (несостоявшимися пересечениями) сверхтонких подуровней. Отметим, недавно мы продемонстрировали, что можно выполнить расчеты по теории кристаллического поля для позиций с точечной группой симметрии $C_{1}$ в $\mathrm{Y}_{2} \mathrm{SiO}_{5}[5]$ и использовать их предсказательную способность для интерпретации спектров со сверхтонкой структурой [6].

Исследование магнитной сверхтонкой структуры в кристаллах, легированных ионами $\mathrm{Eu}^{3+}$ и $\mathrm{Er}^{3+}$, требует применения лазерной спектроскопии высокого разрешения [7]. С другой стороны, ион $\mathrm{Ho}^{3+}$ имеет большой ядерный магнитный момент, и сверхтонкие расщепления могут быть исследованы с использованием обычных методов. Самые ранние оптические исследования сверхтонкой структуры $\mathrm{Ho}^{3+}$ относятся к пионерским работам Герхарда Дике в Университете Джона Хопкинса в середине 1960-х годов. Это исследование с использованием пятиметрового вакуумного спектрометра, изготовленного по заказу фирмой JarrellAsh, показало полностью разрешенную сверхтонкую структуру и зеемановские расщепления для перехода $Z_{1} \rightarrow J_{1}$ на длине волны $23918 \mathrm{~cm}^{-1}$ в кристалле $\mathrm{LaCl}_{3}[8,9]$. В данной работе мы представляем зеемановскую спектроскопию ионов $\mathrm{Ho}^{3+}$ в центре $C_{4 v}\left(\mathrm{~F}^{-}\right)$ в кристалле $\mathrm{CaF}_{2}$, также используя обычную спектроскопию поглощения. Ранее мы сообщали о наблюдении чрезвычайно сложной сверхтонкой структуры, возникающей в результате магнитного взаимодействия двух синглетов в непосредственной близости от орбитального дублета в возбужденном состоянии, сопряженной 
со сверхтонкой структурой основного состояния, обусловленной двумя близко расположенными синглетами, связанными псевдоквадрупольным взаимодействием [1]. В той работе мы получили отличное согласие между теорией и экспериментальными данными как для уровней энергии, так и для интенсивностей переходов, измеренных без приложения магнитного поля. Здесь мы демонстрируем способность моделирования на основе теории кристаллического поля предсказывать зеемановские расщепления сверхтонких состояний и интенсивности переходов, включая наличие антипересечений - эффекта, на котором основана технология ZEFOZ, применяемая в устройствах квантовой информатики.

\section{Эксперимент}

Кристаллы фторида кальция, содержащие 0.01 молярных процента $\mathrm{HoF}_{3}$, были выращены в вакууме по методу Бриджмена-Стокбаргера в высокочастотной печи мощностью 38 kW. Кристаллическую затравку помещали в графитовый тигель (вместе с небольшим количеством $\mathrm{PbF}_{2}$, который действует как абсорбент кислорода) и в течение пяти дней пропускали через температурный градиент, создаваемый рабочей катушкой печи. Кристалл был расколот по плоскости $\langle 111\rangle$, чтобы обеспечить желаемую ориентацию в магнитном поле. Толщина образца для измерения спектра поглощения составляла примерно $5 \mathrm{~mm}$.

Инфракрасные спектры регистрировали с использованием фурье-спектрометра Bruker Vertex 80 (разрешение $0.075 \mathrm{~cm}^{-1}$ ) с оптическим путем, продуваемым газом $\mathrm{N}_{2}$. Зеемановская спектроскопия проводилась с использованием простого соленоида с индукцией магнитного поля 4Т. Сверхпроводящий магнит с образцом, установленным в медный кристаллодержатель, проходящий через центр соленоида, помещался в ванну с жидким гелием. Измерения проводились при температуpe $4.2 \mathrm{~K}$, поскольку медный держатель для образцов находился в прямом тепловом контакте с жидким гелием.

\section{Теоретические основы}

Гамильтониан для моделирования электронной конфигурации $4 f^{\mathrm{N}}$, имеет вид [10-13]

$$
H=H_{\mathrm{FI}}+H_{\mathrm{CF}}+H_{\mathrm{HF}}+H_{Z}
$$

Члены этого уравнения представляют собой гамильтониан свободного иона, взаимодействие с кристаллическим полем, электронно-ядерное сверхтонкое взаимодействие и зеемановское взаимодействие.

Гамильтониан свободного иона подробно обсуждался в упомянутых выше источниках. Гамильтониан кристал- лического поля имеет вид

$$
H_{\mathrm{CF}}=\sum_{k, q} B_{q}^{k} C_{q}^{(k)},
$$

где $k=2,4,6, q=-k, \ldots, k, B_{q}^{k}-$ параметры кристаллического поля, $C_{q}^{(k)}-$ сферические тензорные операторы. В симметрии $C_{4 v}$ мы можем выбрать ось $z$ так, что она будет осью симметрии четвертого порядка, поэтому только параметры с $q=0, \pm 4$ не равны нулю. Гамильтониан сверхтонкого взаимодействия $H_{\mathrm{HF}}$ подробно обсуждается в работе [1]. Ядро гольмия имеет спин $I=7 / 2$, и электронные состояния связаны с ядерными спинами сверхтонким взаимодействием, давая 8 электронно-ядерных состояний для каждого электронного уровня. Для гольмия магнитное сверхтонкое взаимодействие намного больше, чем квадрупольное взаимодействие, но оба они учитываются в расчетах. Важно отметить, что для синглетных электронных состояний диагональные матричные элементы сверхтонких операторов равны нулю, поэтому расщепления являются результатом взаимодействия с соседними электронными состояниями.

Влияние внешнего магнитного поля дается гамильтонианом Зеемана:

$$
H_{Z}=\mu_{\mathrm{B}} \mathbf{B}(\mathbf{L}+2 \mathbf{S}),
$$

где $\mathbf{B}$ - магнитная индукция.

$\mathrm{CaF}_{2}$ - это кубический кристалл. В исследуемых нами центрах симметрии $C_{4 v}$ ион $\mathrm{Ca}^{2+}$ заменен на ион $\mathrm{Ho}^{3+}$ с ближайшим соседним межузельным ионом $\mathrm{F}^{-}$, обеспечивающим компенсацию заряда (в литературе он обычно обозначается как центр $\left.C_{4 v}\left(\mathrm{~F}^{-}\right)\right)$[1]. Ось симметрии четвертого порядка может быть направлена вдоль любой из трех осей четвертого порядка кристалла. В данной работе мы ориентируем кристалл так, чтобы приложить магнитное поле вдоль оси $\langle 111\rangle$. Таким образом, магнитное поле направлено вдоль пространственной диагонали кристалла и одинаково наклонено под углом $\arccos (1 / \sqrt{3})=54.7^{\circ}$ к трем ориентациям центров симметрии $C_{4 v}$. В результате каждый центр испытывает одинаковое магнитное расщепление, что значительно упрощает спектры. Магнитное поле также понижает точечную группу симметрии $\mathrm{Ho}^{3+}$ до $C_{1}$.

В нашей предыдущей работе [1] мы провели детальное исследование с использованием лазерной и инфракрасной спектроскопии. Наше моделирование с использованием теории кристаллического поля дало отличное согласие с экспериментальными значениями электронных и сверхтонких энергий. Были также рассчитаны интенсивности магнитных и электрических дипольных переходов [14], снова в отличном согласии с экспериментальными данными. Здесь мы используем те же параметры кристаллического поля и интенсивностей переходов, что и в упомянутых работах $[1,14]$. Единственным дополнением к гамильтониану является зеемановское взаимодействие (3). Теоретические спектры генерируются с использованием лоренцевой формы линии 

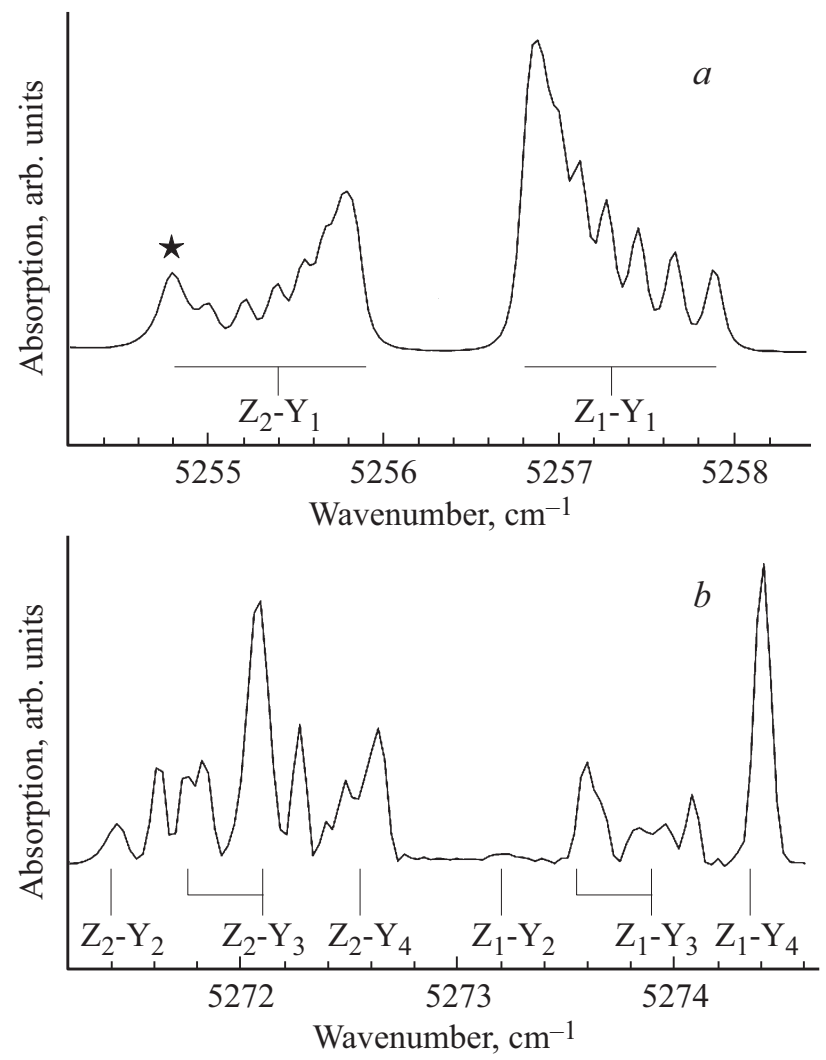

Pис. 1. Спектры при температуре $4.2 \mathrm{~K}$ в нулевом магнитном поле: $a$ - для переходов из $Z_{1} \gamma_{1}, Z_{2} \gamma_{2}\left({ }^{5} I_{8}\right)$ в $\mathrm{Y}_{1} \gamma_{5}\left({ }^{5} I_{7}\right)$, $b$ - для переходов из $Z_{1} \gamma_{1}, Z_{2} \gamma_{2}\left({ }^{5} I_{8}\right)$ в состояния $\mathrm{Y}_{2} \gamma_{3}, \mathrm{Y}_{3} \gamma_{5}$, $\mathrm{Y}_{4} \gamma_{2}\left({ }^{5} I_{7}\right)$. Обозначенная звездочкой линия при $5354.8 \mathrm{~cm}^{-1}$ представляет собой линию поглощения воды $\mathrm{H}_{2} \mathrm{O}$ в атмосфере.

с полушириной $0.09 \mathrm{~cm}^{-1}$. Относительная заселенность состояний $Z_{1} \gamma_{1}$ и $Z_{2} \gamma_{2}$ мультиплета ${ }^{5} I_{8}$ рассчитывалась в соответствии с распределением Больцмана.

\section{Результаты и обсуждение}

Уровни основного состояния центра $C_{4 v}\left(\mathrm{~F}^{-}\right)$в $\mathrm{CaF}_{2}: \mathrm{Ho}^{3+}$ состоят из двух синглетов, разделенных расстоянием $1.7 \mathrm{~cm}^{-1}[15,16]$. Как синглеты (преобразующиеся по неприводимые предтавления $\gamma_{1}$ и $\gamma_{2}$ точечной группы симметрии $C_{4 v}$ ) эти состояния не обладают магнитным моментом. Однако они могут получить его посредством псевдоквадрупольного взаимодействия (и сопутствующего смешивания $\left.A_{8} I_{z} J_{z}\right)$ между ними. На рис. $1, a$ показан низкотемпературный $(4.2 \mathrm{~K})$ и без магнитного поля спектр переходов с этих уровней основного состояния на нижний уровень мультиплета ${ }^{5} I_{7}$ с энергией примерно $5257 \mathrm{~cm}^{-1}$, который представляет собой орбитальный дублет $\left(\mathrm{Y}_{1} \gamma_{5}\right)$. Распределение интенсивности между уровнями определяется смешиванием волновых функций и распределением Больцмана между сверхтонкими уровнями [1]. На рис. $1, b$ показан спектр переходов при температуре $4.2 \mathrm{~K}$ при нулевом магнитном поле с псевдодублета основного состояния
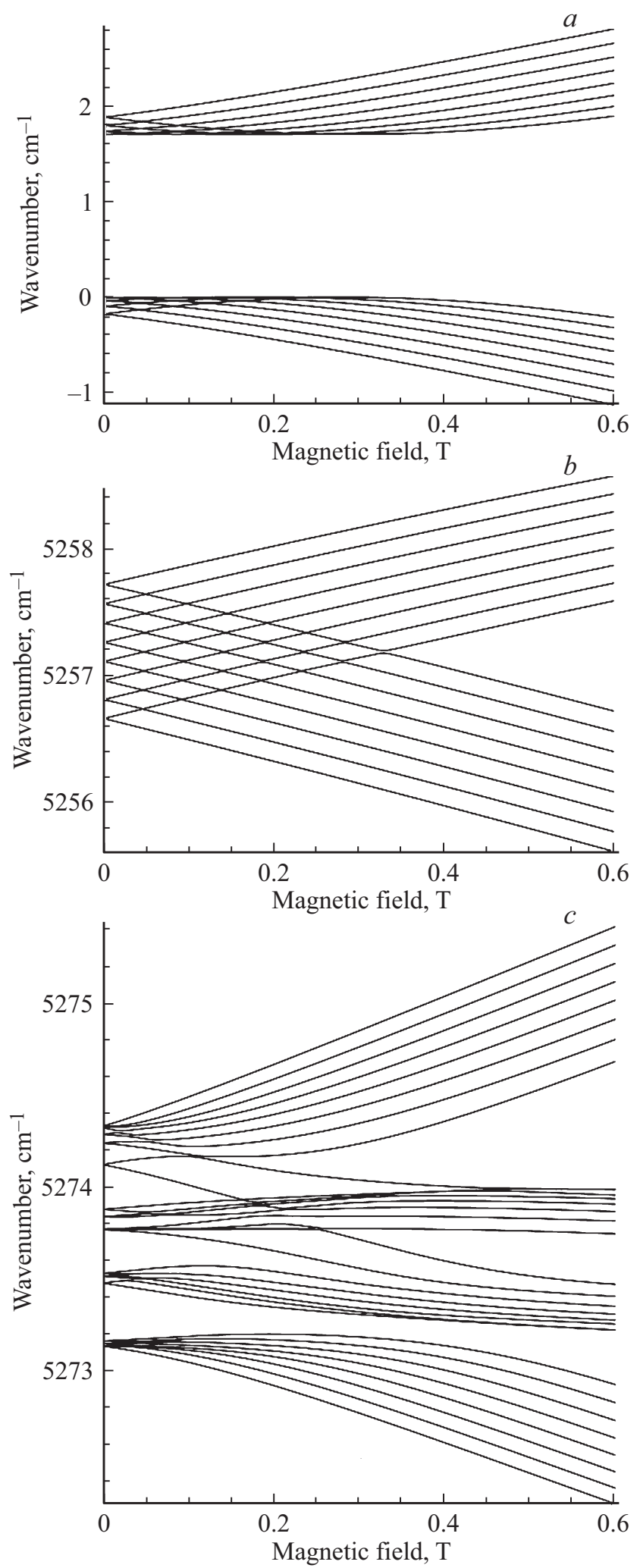

Рис. 2. Расчетные зеемановские сверхтонкие энергии для магнитного поля вдоль направления $\langle 111\rangle$ : для $a-Z_{1} \gamma_{1}, Z_{2} \gamma_{2}$ из ${ }^{5} I_{8}, b-\mathrm{Y}_{1} \gamma_{5}$ из ${ }^{5} I_{7}, c-\mathrm{Y}_{2} \gamma_{3}, \mathrm{Y}_{3} \gamma_{5}, \mathrm{Y}_{4} \gamma_{2}$ из ${ }^{5} I_{7}$.

на другой дублетный уровень возбужденного состояния $\left(\mathrm{Y}_{3} \gamma_{5}\right)$ с энергией $5273.9 \mathrm{~cm}^{-1}$. В этом случае спектр 

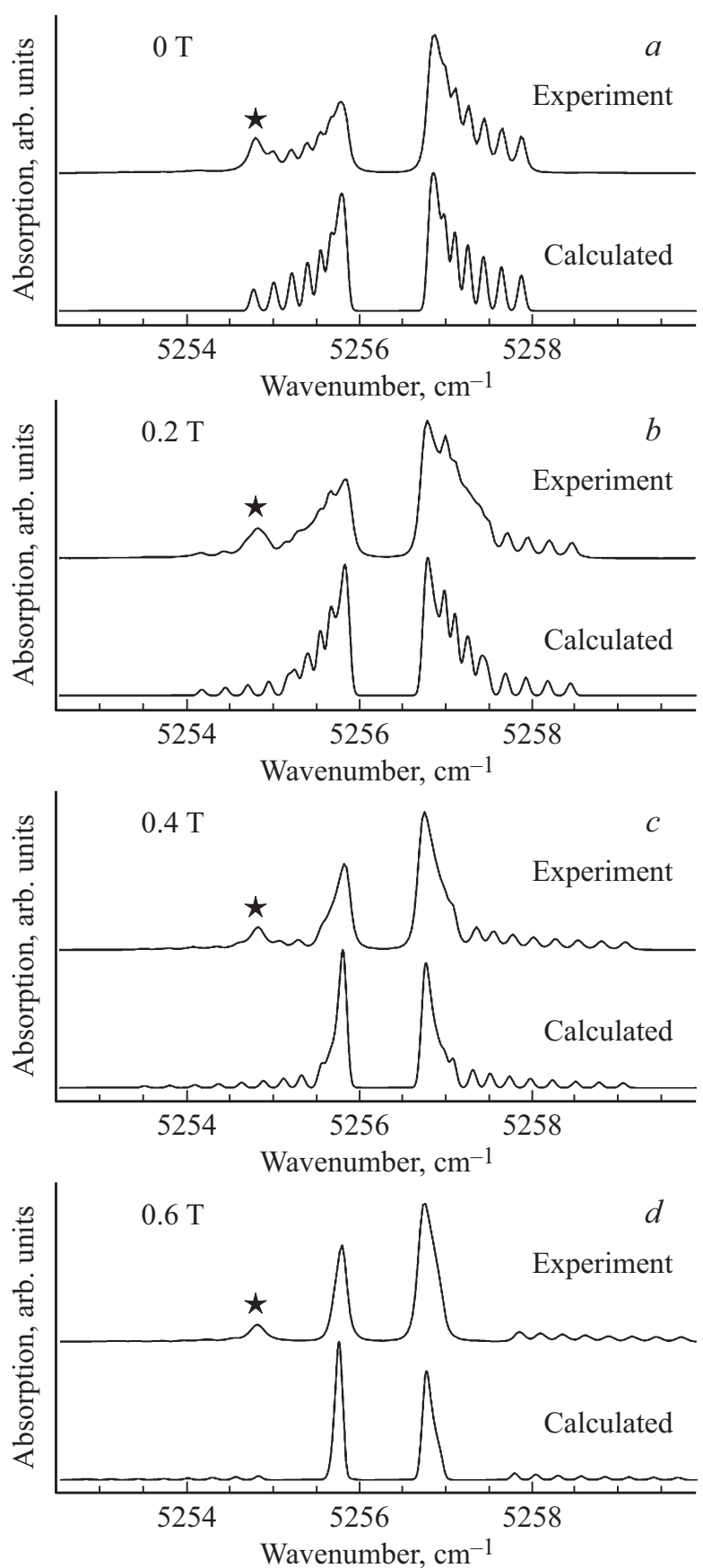

Рис. 3. Экспериментальные и расчетные зеемановские спектры при температуре $4.2 \mathrm{~K}$ для переходов $Z_{1,2} \gamma_{1,2} \rightarrow \mathrm{Y}_{1} \gamma_{5}$ с магнитным полем, направленным вдоль $\langle 111\rangle: a-0.0, b-$ $0.2, c-0.4, d-0.6 \mathrm{~T}$

чрезвычайно сложен. Это происходит из-за непосредственной близости к конечному уровню перехода двух синглетов $\left(\mathrm{Y}_{2} \gamma_{3}\right.$ с энергией $5273.1 \mathrm{~cm}^{-1}$ и $\mathrm{Y}_{4} \gamma_{2}$ с энергией $\left.5274.4 \mathrm{~cm}^{-1}\right)$. Таким образом, наблюдаемая картина возникает из-за смешивания синглетных состояний с дублетом посредством недиагонального сверхтонкого $\left(A_{\perp} 1 / 2\left(I_{+} J_{-}+I_{-} J_{+}\right)\right)$взаимодействия. Из-за сильного смешивания волновых функций происходит значительное перераспределение интенсивностей, и $I_{z}$ перестает быть „хорошим“ квантовым числом, правило отбора $\Delta I_{z}=0$ нарушается. Электронные правила отбора также перестают работать. В частности, переходы $Z_{1} \gamma_{1} \rightarrow \mathrm{Y}_{2} \gamma_{3}$ становятся разрешенными за счет смешивания посредством сверхтонкого взаимодействия.

На рис. 2, a показаны рассчитанные зеемановские расщепления для псевдодублета основного состояния в магнитном поле вдоль направления $\langle 111\rangle$. Это расщепление определяется квадратичным эффектом Зеемана между синглетами, они отталкиваются друг от друга в приложенном поле. Наши расчеты согласуются с измерениями ЭПР, давая $g_{\|}=14.8\left(g_{\perp}=0\right)$ [17]. На рис. $2, b$ и $c$ показаны рассчитанные зеемановские расщепления в магнитном поле вдоль $\langle 111\rangle$ для изолированного дублета $\mathrm{Y}_{1}$ и более сложной псевдоквадруплетной структуры, содержащей состояния $\mathrm{Y}_{2,3,4}$. В первом случае, как и следовало ожидать, имеем линейный эффект Зеемана, тогда как во втором - сильно нелинейные расщепления. В отсутствие сверхтонкого взаимодействия два синглета не взаимодействовали бы друг с другом, и картина полностью определялась бы двумя эффектами: (i) эффектом Зеемана первого порядка в дублете и (ii) квадратичным эффектом Зеемана между дублетом и двумя синглетами. Однако недиагональное сверхтонкое взаимодействие сильно смешивает волновые функции, что изменяет характер электронных уровней, добавляя сложности расщеплениям.

В более общем смысле, именно чрезвычайная чувствительность этих эффектов делает эту систему полезной в качестве теста на предсказательную способность модели кристаллического поля.
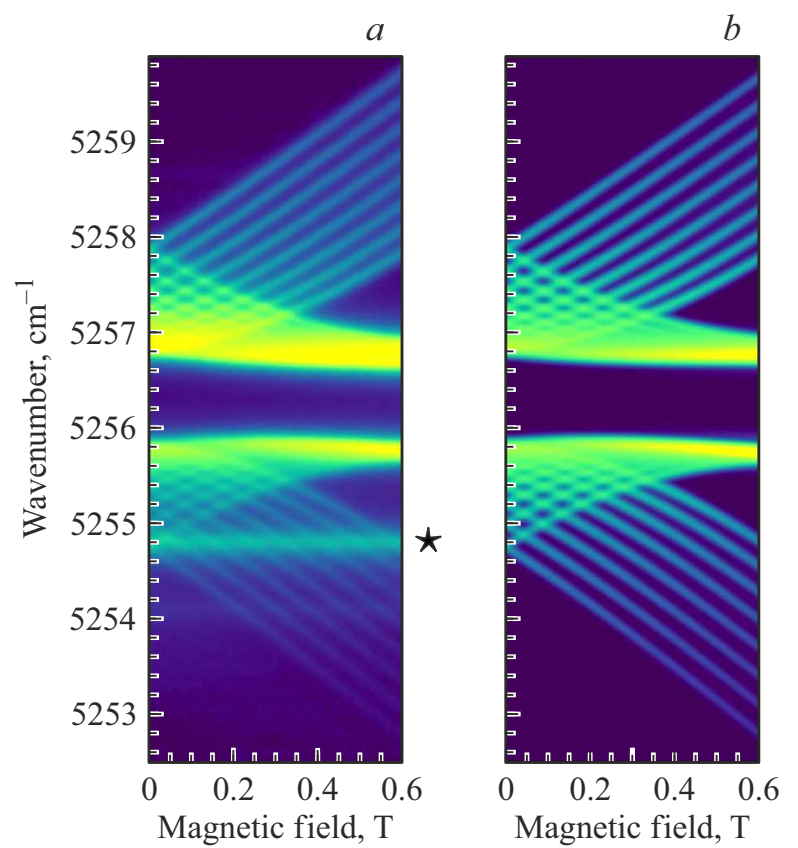

Рис. 4. Карты интенсивности экспериментальных и расчетных зеемановских спектров при температуре $4.2 \mathrm{~K}$ для переходов $Z_{1,2} \gamma_{1,2} \rightarrow \mathrm{Y}_{1} \gamma_{5}$ в магнитных полях, направленных вдоль направления $\langle 111\rangle$ до $0.6 \mathrm{~T}$. 
Экспериментальные и расчетные зеемановские спектры для переходов $Z_{1,2} \gamma_{1,2} \rightarrow \mathrm{Y}_{1} \gamma_{5}$ приведены на рис. 3 для $0.0,0.2,0.4$ и $0.6 \mathrm{~T}$, а экспериментальные и расчетные карты интенсивности от 0.0 до $0.6 \mathrm{~T}$ представлены на рис. 4. Эти переходы аналогичны переходу синглетдублет, показанному на рис. 1 и рис. 3 работы [18]. Однако бо́льшие сверхтонкие и магнитные расщепления нашего синглетного состояния делают наши спектры более сложными.

Приложение магнитного поля вдоль направления $\langle 111\rangle$ понижает симметрию до $C_{1}$, и в принципе все переходы должны быть разрешены. Однако переходы, нарушающие правило отбора $\Delta I_{z}=0$, не наблюдаются, и разрешенные переходы показаны в табл. 8 работы [1]. При увеличении магнитного поля электронный дублет $\mathrm{Y}_{1} \gamma_{5}$ расщепляется. Переходы с основного уровня $Z_{1} \gamma_{1}$ на верхнюю компоненту расщепившегося дублета $\mathrm{Y}_{1} \gamma_{5}$ растут по энергии, тогда как энергия переходов на нижнюю компоненту $\mathrm{Y}_{1} \gamma_{5}$ почти не меняется, поскольку в этом случае начальные и конечные уровни переходов

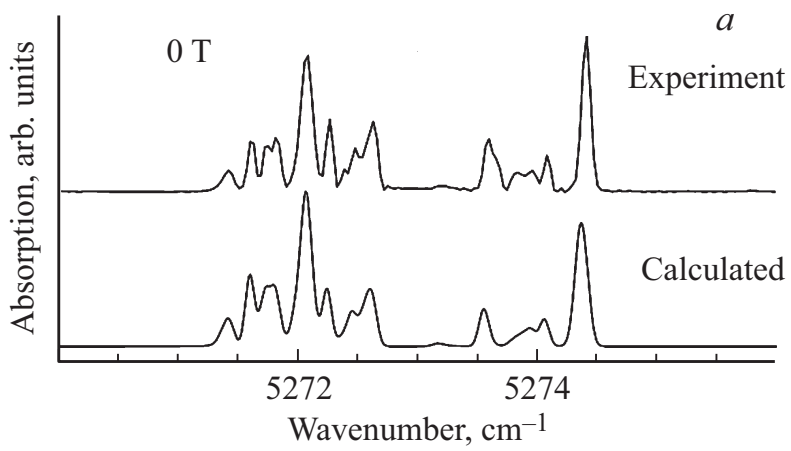

$b$
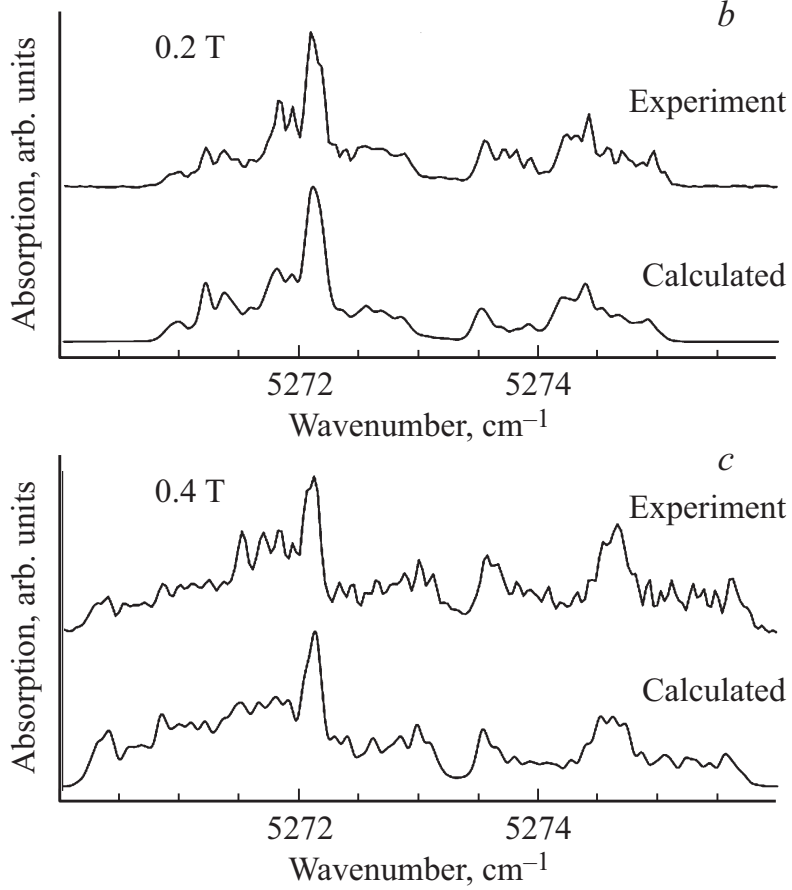

Рис. 5. Экспериментальные и расчетные зеемановские спектры при температуре $4.2 \mathrm{~K}$ для переходов $Z_{1,2} \gamma_{1,2} \rightarrow \mathrm{Y}_{2,3,4} \gamma_{3,5,2}$ с магнитным полем вдоль $\langle 111\rangle: a-0.0, b-0.2, c-0.4 \mathrm{~T}$.

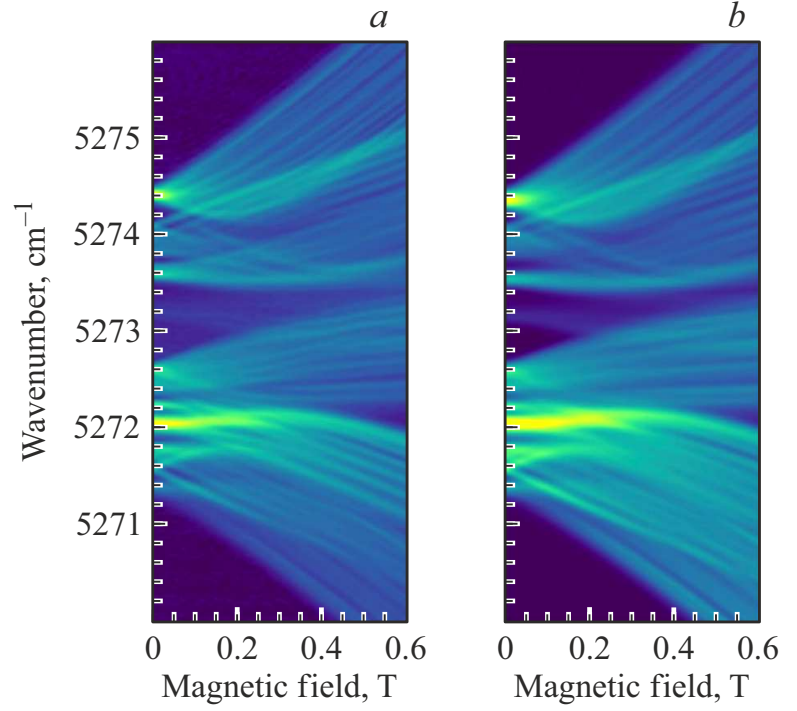

Рис. 6. Карты интенсивности экспериментальных и расчетных зеемановских спектров при температуре $4.2 \mathrm{~K}$ для переходов $Z_{1,2} \gamma_{1,2} \rightarrow \mathrm{Y}_{2,3,4} \gamma_{3,5,2}$ в магнитных полях, направленных вдоль направления $\langle 111\rangle$ до $0.6 \mathrm{~T}$.

сдвигаются почти одинаково в зависимости от магнитного поля (рис. 2, $a$ и $b$ ). Расчеты превосходно согласуются с экспериментом не только по энергиям переходов, но и по их интенсивностям.

Экспериментальные и расчетные зеемановские спектры для переходов $Z_{1,2} \gamma_{1,2} \rightarrow \mathrm{Y}_{2,3,4} \gamma_{3,5,2}$ приведены на рис. 5 для магнитного поля в $0.0,0.2$ и $0.4 \mathrm{~T}$, а экспериментальные и расчетные карты интенсивности от 0.0 до $0.6 \mathrm{~T}$ показаны на рис. 6. Мы приводим данные для сравнительно слабого поля (измерения проводились в полях до 4Т), чтобы проиллюстрировать эффект, поскольку при значительно более сильных полях измеренный спектр слишком широк, чтобы наблюдать детали, а интенсивность значительно снижается. Как можно увидеть на любом из рисунков, наблюдается удивительно хорошее согласие между смоделированными спектрами и экспериментальными данными как по энергиям, так и по интенсивностям переходов.

На рис. 7 показаны рассчитанные антипересечения в состояниях $Z_{1} \gamma_{1}$ и $\mathrm{Y}_{1} \gamma_{5}$, увеличенные с рис. 2, $a$ и $b$. Наибольшая щель в антипересечении для $\mathrm{Y}_{1} \gamma_{5}$ сопоставима с таковыми на рис. 3 работы $[18]\left(0.06 \mathrm{~cm}^{-1}\right)$. Однако бо́льшая ширина линий в $\mathrm{CaF}_{2}$ по сравнению c $\mathrm{LiYF}_{4}$ может затруднить разрешение антипересечений даже для кристаллов с низкой концентрацией редкоземельных ионов и при использовании более высокого спектрального разрешения, такого как в спектрах на рис. 5 в работе [1]. Антипересечения в $Z_{1} \gamma_{5}$ составляют порядка 70-150 MHz и потенциально могут быть исследованы на радиочастотах. В работе [19] для гольмия в молекулярном магнетике в экспериментах по импульсному ЭПР было продемонстрировано увеличение времени когерентности в точках антипересечений между сверхтонкими уровнями электронных состояний $Z_{1}$ и 

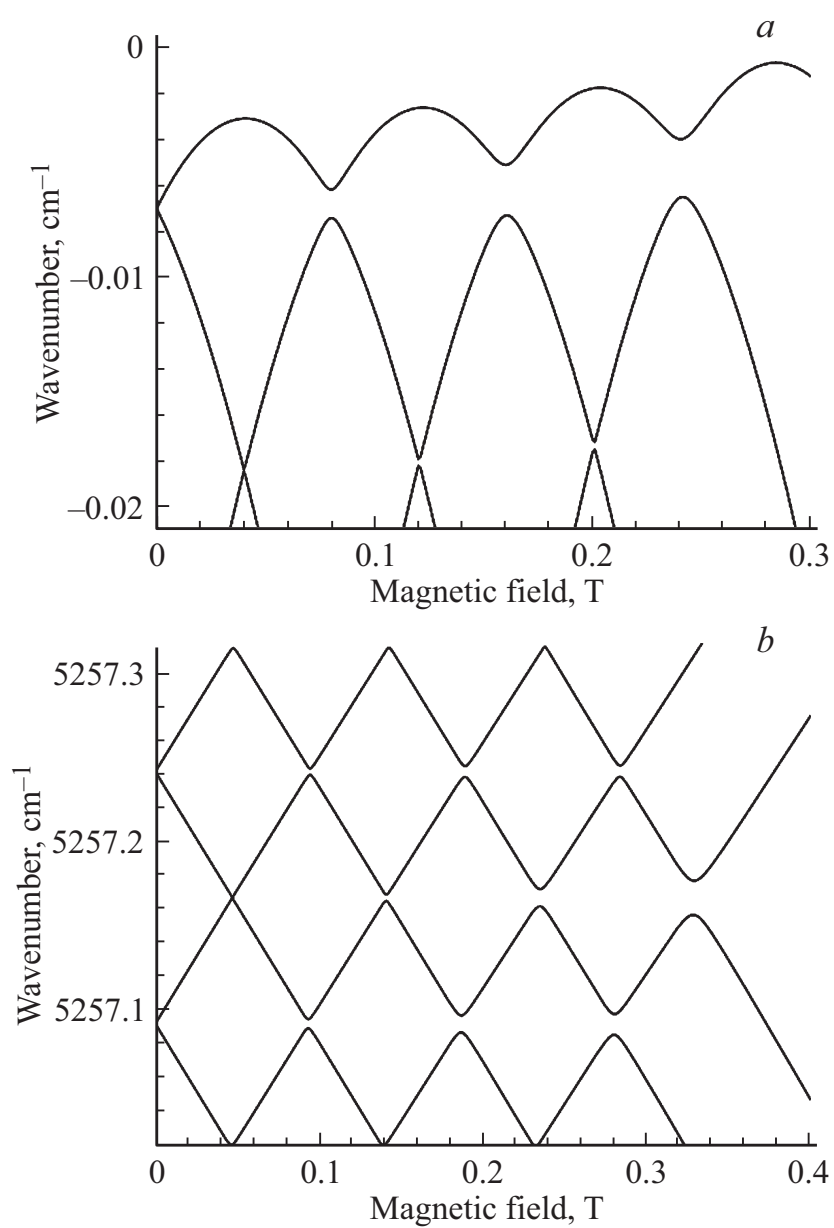

Рис. 7. Расширенные расчеты уровней энергии с магнитным полем, направленным вдоль направления $\langle 111\rangle$ для: $a-Z_{1} \gamma_{1}$ из ${ }^{5} I_{8}$ и $b-\mathrm{Y}_{1} \gamma_{5}$ из ${ }^{5} I_{7}$.

$Z_{2}$ иона гольмия. Однако расщепление между $Z_{1} \gamma_{1}$ и $Z_{2} \gamma_{2}$ в нашем случае намного больше $(50.8 \mathrm{GHz})$, чем расщепление, используемое в экспериментах ЭПР $(9 \mathrm{GHz})$. В состояниях $\mathrm{Y}_{2} \gamma_{3}, \mathrm{Y}_{3} \gamma_{5}, \mathrm{Y}_{4} \gamma_{2}$ явно имеются многочисленные антипересечения. Однако сложность спектров чрезвычайно затрудняет определенную идентификацию.

\section{Заключение}

Исследована сверхтонкая структура в спектре иона $\mathrm{Ho}^{3+}$ в центре $C_{4 v}\left(\mathrm{~F}^{-}\right)$в кристалле $\mathrm{CaF}_{2}$, находящемся в магнитном поле, приложенном вдоль кристаллографического направления $\langle 111\rangle$. В частности, представлены результаты для переходов на изолированный орбитальный дублет и псевдоквадруплетную группировку уровней, состоящую из дублета и двух близлежащих синглетов. Модель кристаллического поля, разработанная ранее в работе [1] с добавлением только зеемановского члена к гамильтониану, объясняет экспериментальные энергии и интенсивности переходов с поразительной точностью. Это сулит хороший результат для более сложной задачи прогнозирования точек ZEFOZ (значений магнитного поля, при которых эффект Зеемана первого порядка отсутствует) в материалах с низкой симметрией, используемых для хранения квантовой информации.

\section{Конфликт интересов}

Авторы заявляют, что у них нет конфликта интересов.

\section{Список литературы}

[1] J.-P.R. Wells, G.D. Jones, M.F. Reid, M.N. Popova, E.P. Chukalina. Mol. Phys., 102, 1367 (2004).

[2] M. Zhong, M.P. Hedges, R.L. Ahlefeldt, J.G. Bartholomew, S.E. Beavan, S.M. Wittig, J.J. Longdell, M.J. Sellars. Nature, 517, 177 (2015).

[3] Y. Ma, Y.-Z. Ma, Z.-Q. Zhou, C.-F. Li, G.-C. Guo. Nature Communications, 12, 2381 (2021).

[4] M. Rančić, M.P. Hedges, R.L. Ahlefeldt, M.J. Sellars. Nature Physics, 14, 50 (2018).

[5] S.P. Horvath, J.V. Rakonjac, Y.-H. Chen, J.J. Longdell, P. Goldner, J.P.R. Wells, M.F. Reid. Phys. Rev. Lett., 123, 057401 (2019).

[6] S. Mothkuri, M.F. Reid, J.-P.R. Wells, E. Lafitte-Houssat, P. Goldner, A. Ferrier. Phys. Rev. B, 103, 104109 (2021).

[7] R.M. Macfarlane, R.M. Shelby. In Spectroscopy of Solids Containing Rare Earth Ions, ed. by A.A. Kaplyanskii, R.M. Macfarlane (Elsevier Science Publishers B.V., Amsterdam, 1987).

[8] G.H. Dieke, B. Pandey. J. Chem. Phys., 41, 1952 (1964).

[9] H.M. Crosswhite, H.W. Moos. In Optical Properties of Ions in Crystals, ed. by H.M. Crosswhite, H.W. Moos (Interscience, New York, 1967).

[10] W.T. Carnall, G.L. Goodman, K. Rajnak, R.S. Rana. J. Chem. Phys., 90, 3443 (1989).

[11] C. Görller-Walrand, K. Binnemans. In Handbook on the Physics and Chemistry of Rare Earths, ed. by J.K.A. Gschneidner, L. Eyring (North-Holland, Amsterdam, 1996) v. 23, p. 121.

[12] G. Liu. In Spectroscopic Properties of Rare Earths in Optical Materials, ed. by G. Liu, B. Jacquier (Springer Science \& Business Media, 2006).

[13] M.F. Reid. In Handbook on the Physics and Chemistry of Rare Earths, ed. by J.-C. Bünzli, V.K. Pecharsky (North Holland, Amsterdam 2016), v. 50, p. 47-64.

[14] M.F. Reid. In Spectroscopic Properties of Rare Earths in Optical Materials, ed. by G. Liu, B. Jacquier (Springer Science \& Business Media, 2006).

[15] M. Mujaji, G.D. Jones, R.W.G. Syme. Phys. Rev. B., 46, 14398 (1992).

[16] J.P.D. Martin, T. Boonyarith, N.B. Manson, M. Mujaji, G.D. Jones. J. Phys.: Cond. Matt., 5, 1333 (1993).

[17] L.S. Kornienko, A.A. Rybaltovskii. Sov. Phys. Sol. St., 13, 1785 (1972).

[18] K.N. Boldyrev, M.N. Popova, B.Z. Malkin, N.M. Abishev. Phys. Rev. B, 99, 041105(R) (2019).

[19] M. Shiddiq, D. Komijani, Y. Duan, A. Gaita-Ariño, E. Coronado, S. Hill. Nature, 531, 348 (2016).

Перевод с английского А. Яблуновский 